\title{
Colorful Paths in Vertex Coloring of Graphs
}

\author{
Saieed Akbari* \\ Department of Mathematical Sciences, \\ Sharif University of Technology, \\ Tehran, Iran \\ School of Mathematics, \\ Institute for Research in Fundamental Sciences(IPM), \\ Tehran, Iran \\ s_akbari@sharif .edu \\ Vahid Liaghat \\ Computer Engineering Department, \\ Sharif University of Technology, \\ Tehran, Iran \\ liaghat@ce.sharif .edu \\ Afshin Nikzad \\ Computer Engineering Department, \\ Sharif University of Technology, \\ Tehran, Iran \\ nikzad@ce.sharif.edu
}

Submitted: Nov 16, 2009; Accepted: Dec 22, 2010; Published: Jan 12, 2011 Mathematics Subject Classification: 05C15

\begin{abstract}
A colorful path in a graph $G$ is a path with $\chi(G)$ vertices whose colors are different. A $v$-colorful path is such a path, starting from $v$. Let $G \neq C_{7}$ be a connected graph with maximum degree $\Delta(G)$. We show that there exists a $(\Delta(G)+1)$-coloring of $G$ with a $v$-colorful path for every $v \in V(G)$. We also prove that this result is true if one replaces $(\Delta(G)+1)$ colors with $2 \chi(G)$ colors. If $\chi(G)=\omega(G)$, then the result still holds for $\chi(G)$ colors. For every graph $G$, we show that there exists a $\chi(G)$-coloring of $G$ with a rainbow path of length $\lfloor\chi(G) / 2\rfloor$ starting from each $v \in V(G)$.
\end{abstract}

Keywords: Vertex-coloring, Colorful path, Rainbow path

\section{Introduction}

Throughout this paper all graphs are simple. Let $G$ be a graph and $V(G)$ be the vertex set of $G$. In a connected graph $G$, for any two vertices $u, v \in V(G)$ let $d_{G}(u, v)$ denote the

${ }^{*}$ Corresponding author. S. Akbari 
length of the shortest path between $u$ and $v$ in $G$. We denote the DFS tree in $G$ rooted at $v$ by $T(G, v)$ (which is defined in [2, p.139]). For every $u \in V(G)$, each vertex on the path between $u$ and $v$ in $T(G, v)$ is called an ancestor of $u$. By Theorem 6.6 of [2, in every DFS tree if $w$ and $w^{\prime}$ are adjacent, then one of them is ancestor of another.

In a graph $G$, a $k$-coloring of $G$ is a function $c: V(G) \rightarrow\{0, \ldots, k-1\}$ such that $c(u) \neq c(v)$ for every adjacent vertices $u, v \in V(G)$. The chromatic number of $G$ denoted by $\chi(G)$, is the smallest $k$ for which $G$ has a $k$-coloring. For simplicity we denote a $\chi(G)$-coloring of $G$ by $\chi$-coloring. For a coloring of graph $G$, we say path $P$ of $G$ is a rainbow path if all vertices of $P$ have different colors. A $v$-rainbow path is a rainbow path starting from the vertex $v$. A $v$-colorful path is a rainbow path starting from the vertex $v$ with $\chi(G)$ vertices. The colorful paths and rainbow paths have been studied by several authors, see [4], 5] and [6].

For each $u \in V(G)$, let $N(u)$ be the set of all vertices adjacent to $u$. We denote a cycle of order $n$ by $C_{n}$. Also we denotes the size of the maximum clique in $G$ by $\omega(G)$. A good cycle in a graph $G$ is a cycle of order $\ell$ in which $\ell \geq \chi(G)$ and $\ell=0$ or $\ell=1(\bmod \chi(G))$.

\section{The Existence of $(\Delta(G)+1)$-Colorings with Colorful Paths}

Let $G$ be a graph. We recall that a path in $G$ is said to represent all $\chi(G)$ colors if all the colors $0, \ldots, \chi(G)-1$ appear on this path. The following problem was posed in 6 .

Problem. Let $G$ be a connected graph. Does there always exist a proper vertex coloring of $G$ with $\chi(G)$ colors such that every vertex of $G$ is on a path with $\chi(G)$ vertices which represents all $\chi(G)$ colors?

The following conjecture was proposed in [1].

Conjecture. Let $G \neq C_{7}$ be a connected graph. Then there exists a $\chi(G)$-coloring of $G$ such that for every $v \in V(G)$, there exists a v-colorful path.

In [1] it is shown that the local version of conjecture is true, that is for an arbitrary $v \in V(G)$, there exists a $\chi$-coloring of $G$ with a $v$-colorful path. We start with the following theorem.

Theorem 1 Let $G \neq C_{7}$ be a connected graph. If $G$ contains a good cycle, then there is $a(\Delta(G)+1)$-coloring of $G$ with a $v$-colorful path for every $v \in V(G)$.

Proof. For complete graphs the assertion is trivial. Fig.1 shows a proper 3-coloring for odd cycles except $C_{7}$, with a $v$-colorful path for every $v \in V(G)$. Thus assume that $G$ is neither an odd cycle nor a complete graph.

Assume that $C$ is a good cycle of the minimum order $k$ in $G$, with vertices $v_{0}, v_{1}, \ldots$, $v_{k-1}$, such that $k=0$ or $k=1(\bmod \chi(G))$. For every $i, 0 \leq i \leq k-1$, we color the 


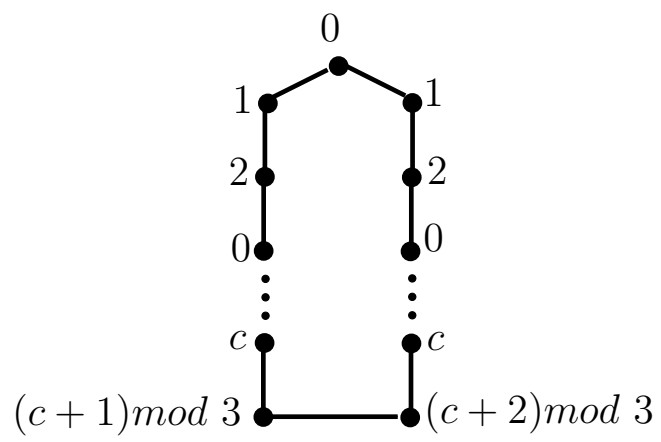

Figure 1: Coloring of odd cycles not isomorphic to $C_{7}$

vertex $v_{i}$ by $i \bmod \chi(G)$ using the colors $0, \ldots, \chi(G)-1$. In the case $k=1(\bmod \chi(G))$, we color $v_{k-1}$ by the color $\chi(G)$ and call $v_{k-1}$ by $v^{*}$. Note that because of the minimality of the order of $C$, there is no edge between two vertices of the same color and for each $i, 0 \leq i \leq k-1$, there is a $v_{i}$-colorful path on $C$. As a consequence of Brooks' Theorem (Theorem 14.4 of [2]), in the coloring of $C$ we use at most $\Delta(G)+1$ colors. For each $i$, $0 \leq i \leq k-1$, let father of $v_{i}$ (for abbreviation $F\left(v_{i}\right)$ ) be $v_{((i+1) \bmod k)}$.

Now, we provide an algorithm to color the remaining vertices of $G$ with $\Delta(G)+1$ colors such that there is a $v$-colorful path for each $v \in V(G)$. For simplicity, define $N$ ext $(t)$ the color $(t+1) \bmod (\Delta(G)+1)$, for every $t, 0 \leq t \leq \Delta(G)$.

In each step of the algorithm, let $u$ be one of the vertices with no color, but adjacent to some colored vertices. Let $c(N(u))$ be the set of all colors appeared in the neighbors of $u$. Since $|c(N(u))| \leq \Delta(G)$, we can choose an available color $t$ such that $t \notin c(N(u))$ but $N \operatorname{ext}(t) \in c(N(u))$.

Let $F(u)$ be one of the vertices in $N(u)$ whose color is $N$ ext $(t)$. Assign the color $t$ to $u$ and continue the algorithm until all vertices are colored.

Obviously the algorithm produces a proper coloring $c$. Now, we show that there is a $u$-colorful path. Consider the following sequence of the vertices $Q(u): q_{1}, \ldots, q_{\chi(G)}$ such that $q_{1}=u$ and for every $i, 1<i \leq \chi(G): q_{i}=F\left(q_{i-1}\right)$. We prove that $Q(u)$ is a $u$-colorful path. We claim that the colors of $q_{1}, \ldots, q_{\chi(G)}$ are distinct.

The proof is by contradiction. It can be easily checked that the following holds:

$$
c\left(q_{i+1}\right)= \begin{cases}c\left(q_{i}\right)+1(\bmod (\Delta(G)+1)) & \text { if } q_{i} \notin C \\ c\left(q_{i}\right)+1(\bmod \chi(G)) & \text { if } q_{i}, q_{i+1} \in C \backslash\left\{v^{*}\right\} \\ c\left(q_{i}\right)+1(\bmod (\chi(G)+1)) & \text { if } q_{i}=v^{*} \text { or } q_{i+1}=v^{*}\end{cases}
$$

Assume that for some $a \neq b, c\left(q_{a}\right)=c\left(q_{b}\right)$. It is clear that for some $i, a \leq i<b$, $c\left(q_{i}\right)=0$. Let $M=\max \left\{i \mid i<b, c\left(q_{i}\right)=0\right\}$. The colors of the vertices $q_{M}, q_{M+1}, \ldots, q_{b}$ are $0,1, \ldots, c\left(q_{b}\right)$, respectively. Since the number of vertices of $Q(u)$ is $\chi(G)$, we have $0<c\left(q_{b}\right)<\chi(G)$. 
Now, let $m=\min \left\{i \mid a<i, c\left(q_{i}\right)=0\right\}$. Since $c\left(q_{a}\right) \neq 0$, we have $m \leq M$. The number of vertices in the sequence $q_{M}, \ldots, q_{b}$ is exactly $c\left(q_{b}\right)+1$. Since $c\left(q_{m}\right)=0$, $c\left(q_{m-1}\right) \in\{\chi(G)-1, \chi(G), \Delta(G)\}$. So the number of vertices in the sequence $q_{a}, \ldots, q_{m-1}$ is at least $\chi(G)-c\left(q_{a}\right)$. Therefore the number of vertices of $Q(u)$ should be at least $\chi(G)+1$, a contradiction. The claim is proved.

Before stating our main results, we need to prove another theorem.

Lemma 1 Let $G$ be a connected graph with no cycle of order $\chi(G)$. For a given vertex $v$, there exists $u \in V(G)$ such that $2 \chi(G)-2 \leq d_{T(G, v)}(u, v)$.

Proof. Let $T=T(G, v)$. If for every $w \in V(G), 2 \chi(G)-2>d_{T}(w, v)$, then we show one can properly color the vertices of $G$ using $\chi(G)-1$ colors. To see this we define a coloring $c$ as follows. For every $w \in V(G)$, let $c(w)=d_{T}(w, v)(\bmod (\chi(G)-1))$. Assume that $w_{1}, w_{2} \in V(G)$ are adjacent and $c\left(w_{1}\right)=c\left(w_{2}\right)$. Since $T$ is a DFS tree, with no loss of generality we can suppose that $w_{1}$ is an ancestor of $w_{2}$. Thus $d_{T}\left(w_{1}, w_{2}\right)=$ $0(\bmod (\chi(G)-1))$. If $d_{T}\left(w_{1}, w_{2}\right) \neq \chi(G)-1$, then $d_{T}\left(w_{2}, v\right) \geq 2 \chi(G)-2$; a contradiction. Hence $d_{T}\left(w_{1}, w_{2}\right)=\chi(G)-1$. Since $w_{1}$ and $w_{2}$ are adjacent we find a cycle of order $\chi(G)$; a contradiction.

The following theorem proves the assertion of Theorem 1 for the graphs with no good cycle.

Theorem 2 Let $G \neq C_{7}$ be a connected graph. If $G$ has no good cycle, then there is a $(\Delta(G)+1)$-coloring of $G$ with a v-colorful path for every $v \in V(G)$.

Proof. As we see in the proof of Theorem 1, the assertion holds for odd cycles except $C_{7}$. Thus assume that $G$ is not an odd cycle. Let $v$ be an arbitrary vertex of $G$ and $T=T(G, v)$. By Lemma 1. there exists a vertex $u$ such that $2 \chi(G)-2 \leq d_{T}(u, v)$. Let $P: v=p_{0}, p_{1}, \ldots, p_{k}=u$ be the path between $v$ and $u$ in $T$. Let $Q$ represent the set of vertices of $G$ whose ancestors(including the vertex itself) are not in the set $\left\{p_{\chi(G)-1}, p_{\chi(G)}, \ldots, p_{k}\right\}$. Define $S=Q \backslash P$ (See Fig.2).

For each $w \in V(G) \backslash S$, color $w$ with $d_{T}(w, v)$ mod $\chi(G)$. Since there are no good cycles in $G$, therefore the coloring of $V(G) \backslash S$ is proper. For each $w \in Q \backslash S$, there is a $w$-colorful path in $V(G) \backslash S$ going downward in $T$ through $P$, by passing from each vertex to its child in $P$. For each $w \in V(G) \backslash Q$, there is a $w$-colorful path in $V(G) \backslash S$ going upward in $\mathrm{T}$ by passing from each vertex to its parent.

So for each $w \in V(G) \backslash S$, there is a $w$-colorful path. All uncolored vertices are contained in $S$. We color them in such a way that for each $w \in S$ there exists a vertex $w^{\prime} \in N(w)$, where $c\left(w^{\prime}\right)=N \operatorname{ext}(c(w))$. Recall that for a color $t, N \operatorname{ext}(t)=$ $(t+1) \bmod (\Delta(G)+1)$. We denote $w^{\prime}$ by $F(w)$. Since $T$ is a DFS tree there are no edges between $S$ and $V(G) \backslash Q$. Therefore $F(w) \in Q$. Such coloring can be obtained using the algorithm discussed in the proof of Theorem 1. Now, we show that for each $w \in S$, there exists a $w$-colorful path. 


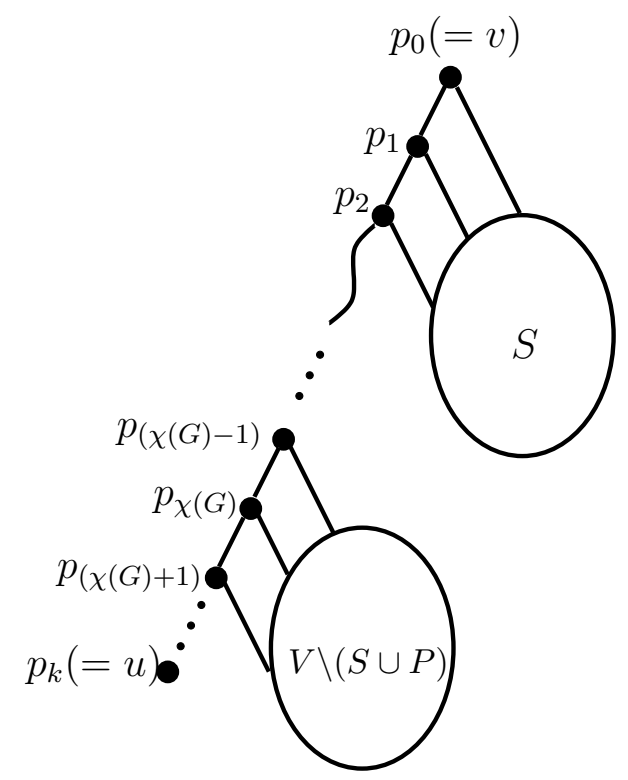

Figure 2: The DFS tree $T$, rooted at $v$. This figure illustrates only the edges of $T$.

For every $i, 0 \leq i \leq k-1$, let $F\left(p_{i}\right)=p_{i+1}$. Consider the sequence of the vertices $Q(w): q_{0}(=w), \ldots, q_{\chi(G)-1}$, where $F\left(q_{i}\right)=q_{i+1}$, for every $i, 0 \leq i<\chi(G)-1$. Note that for each $i, 0 \leq i<\chi(G)-1, c\left(q_{i+1}\right)$ is either $N \operatorname{ext}\left(c\left(q_{i}\right)\right)$ or $c\left(q_{i}\right)+1(\bmod \chi(G))$. Hence there are no vertices with the same color in $Q(w)$. Therefore $Q(w)$ is a $w$-colorful path.

The following theorem shows that for every graph $G$ the conjecture is true for $\Delta(G)+1$ colors instead of $\chi(G)$ colors. In [1] it was proved that the conjecture is true for $\chi(G)+$ $\Delta(G)-1$ colors. The following theorem is an improvement of this result.

Theorem 3 Let $G \neq C_{7}$ be a connected graph. Then there is a $(\Delta(G)+1)$-coloring of $G$ with a $v$-colorful path, for every $v \in V(G)$.

Proof. If $G \neq C_{7}$ contains a good cycle, then by Theorem 1 there is a $(\Delta(G)+1)$-coloring of $G$ with a $v$-colorful path, for every $v \in V(G)$. Thus, we may assume that $G$ does not have a good cycle. In this case, Theorem 2 shows that there is a $(\Delta(G)+1)$-coloring of $G$ with the same properties.

\section{The Existence of $(2 \chi(G))$-Colorings with Colorful Paths}

Let $c$ be a $\chi$-coloring of a given graph $G$. Let $G_{c}$ be a directed graph with the same vertex set of $G$ which has a directed edge from $u$ to $v$ if and only if (i) $u$ and $v$ are adjacent in $G$; and $($ ii) $c(v)=c(u)+1(\bmod \chi(G))$. 
Lemma 2 Let $c$ be a $\chi$-coloring of a connected graph $G$. For a given subgraph $H$ of $G$, there exists a $\chi$-coloring $c^{\prime}$, such that for every $v \in V(H), c^{\prime}(v)=c(v)$ and for every $u \in V(G)$, there is a directed path from $u$ to at least one of the vertices of $V(H)$ in $G_{c^{\prime}}$.

Proof. For an arbitrary $\chi$-coloring of $G$ like $c$, a vertex $u$ in $G_{c}$ is called nice if there exists a directed path from $u$ to a vertex of $H$. Assuming that we have a $\chi$-coloring $c$, we give a polynomial-time algorithm to obtain the coloring $c^{\prime}$ from $c$, such that all the vertices are nice. Let $c^{\prime}=c$ and let $S \in V(G)$ be the set of all vertices of $G$ which are not nice in $c^{\prime}$. We will decrease $|S|$, by modifying $c^{\prime}$ in each iteration of the algorithm. After at most $|V|$ iterations, all the vertices would be nice.

In each iteration, we do as follows:

Let $c_{i}^{\prime}$, for $i, 1 \leq i<\chi(G)$, be the coloring of $G$ such that:

$$
c_{i}^{\prime}(v)= \begin{cases}c^{\prime}(v) & \text { if } v \notin S \\ c^{\prime}(v)+i(\bmod \chi(G)) & \text { if } v \in S .\end{cases}
$$

Since $G$ is connected, at least one of these colorings is not proper. Assume that $t$ is the smallest natural number for which $c_{t}^{\prime}$ is not proper. By the definition of $S$, there is no directed edge from $S$ to $V(G) \backslash S$ in $G_{c^{\prime}}$. Hence $c_{1}^{\prime}$ is proper. Now, consider the proper coloring $c_{t-1}^{\prime}$. Since $c_{t}^{\prime}$ is not proper, there are two adjacent vertices $u \in S$ and $v \notin S$ such that $c_{t-1}^{\prime}(u)+1=c_{t-1}^{\prime}(v)(\bmod \chi(G))$. Therefore $u$ is also a nice vertex in $G_{c_{t-1}^{\prime}}$. Now, let $c^{\prime}$ be $c_{t-1}^{\prime}$ and continue with the next iteration (note that the vertices of $G \backslash S$ remain nice in $c^{\prime}$ and $u$ becomes a nice vertex).

After at most $|V|$ iterations the algorithm will find a coloring $c^{\prime}$ such that all vertices are nice, and each iteration can be implemented in $O(|V|+|E|$ ) time (by considering the edges between $S$ and $G \backslash S)$.

We denote the $\chi$-coloring $c^{\prime}$, given in the proof of Lemma 2. by $\mathcal{C}(G, H, c)$. Next theorem shows that for every graph $G$ the conjecture holds if one replaces $\chi(G)$ colors with $2 \chi(G)$ colors.

Theorem 4 Let $G$ be a connected graph. Then there exists a $2 \chi(G)$-coloring of $G$ with a v-colorful path for every $v \in V(G)$.

Proof. Let $H=C$ when there is a cycle $C$ of order $\chi(G)$ or $\chi(G)+1$, otherwise let $H$ be the path with $2 \chi(G)-1$ vertices according to Lemma 1. In either case, choose an arbitrary vertex of $H$ and call it by $v^{*}$. Let $c$ be a $\chi$-coloring of $G$ and set $c^{\prime}=\mathcal{C}\left(G, v^{*}, c\right)$. Now we recolor vertices of $H$ with at most $\chi(G)$ new colors $\chi(G), \ldots, 2 \chi(G)-1$ such that:

- If $H$ is a cycle, then color vertices of $H \backslash v^{*}$ with one of the colors $\chi(G), \ldots, 2 \chi(G)-1$. Color $v^{*}$ as the same as its color in $\mathcal{C}\left(G, v^{*}, C\right)$.

- If $H: p_{0}, \ldots, p_{2 \chi(G)-2}$ is a path, then color $p_{i}$ with $\chi(G)+(i \bmod \chi(G))$. 
We first claim that $c^{\prime}$ is a proper coloring. This is trivial in the first case. In the case $H$ is a path $P$, if there are two adjacent vertices $u, v \in V(G)$ with the same color in $c^{\prime}$, then $u, v \in V(P)$, because $V(G) \backslash H$ is properly colored with the colors $0, \ldots, \chi(G)-1$ and $H$ is colored with the colors $\chi(G), \ldots, 2 \chi(G)-1$. Let $p_{i}=u$ and $p_{j}=v$. With no loss of generality suppose that $i<j$. Note that in the coloring of $P$, we should have $i=j(\bmod \chi(G))$. So the vertices $p_{i}, \ldots, p_{j}$ form a cycle of order $\chi(G)+1$, a contradiction.

Now, we show that for each $v \in V(G)$, there is a $v$-colorful path in $c^{\prime}$.

Case 1. $H$ is a cycle with the vertices $D: v_{0}, \ldots, v_{k}$, where $k=\chi(G)-1$ or $\chi(G)$. Let $v$ be an arbitrary vertex of $G$. If $v \in D$, then it is clear that there is a $v$-colorful path in $D$. If $v \notin D$, then by Lemma 2, there exists a directed path starting from $v$ and ending to $v^{*}$ in $G_{f}$, where $f=\mathcal{C}\left(G, v^{*}, c\right)$. Call this path by $Q: q_{0}(=v), \ldots, q_{k}\left(=v^{*}\right)$. If $k \geq \chi(G)-1$, then $q_{0}, \ldots, q_{\chi(G)-1}$ is a $v$-colorful path. So assume that $k<\chi(G)-1$. Let $i$ be the smallest index such that $q_{i} \in D$. Consider the $q_{i}$-colorful path in $D$ and call it by $Q^{\prime}: q_{0}^{\prime}\left(=q_{i}\right), \ldots, q_{\chi(G)-1}^{\prime}$. We claim that $Q^{\prime \prime}: q_{0}, \ldots, q_{i}, q_{1}^{\prime}, \ldots, q_{\chi(G)-i-1}^{\prime}$ is a $v$-colorful path. The vertices of $D$ are differently colored with the colors $c\left(v^{*}\right), \chi(G), \ldots, 2 \chi(G)-1$. Since $k<\chi(G)-1$, there are no vertices colored with $c\left(v^{*}\right)$ in $\left\{q_{0}, \ldots, q_{i}\right\}$. Therefore $Q^{\prime \prime}$ is a $v$-colorful path.

Case 2. $H$ is a path $P$. Let $v$ be an arbitrary vertex of $G$. If $v \in V(P)$, then according to the length of $P$, there is a $v$-colorful path in $P$. If $v \notin V(P)$, then by Lemma 2 , there is a directed path starting from $v$ and ending to $v^{*}$ in $G_{f}$, where $f=\mathcal{C}\left(G, v^{*}, c\right)$. Call this path by $Q: q_{0}(=v), \ldots, q_{k}\left(=v^{*}\right)$. Let $i$ be the smallest index such that $q_{i} \in V(P)$. If $i \geq \chi(G)-1$, then $q_{0}, \ldots, q_{\chi(G)-1}$ is a $v$-colorful path. If $i<\chi(G)-1$, then consider the $q_{i^{-}}$ colorful path in $P$ and call it by $Q^{\prime}: q_{0}^{\prime}\left(=q_{i}\right), \ldots, q_{\chi(G)-1}^{\prime}$. Then $q_{0}, \ldots, q_{i}, q_{1}^{\prime}, \ldots, q_{\chi(G)-i-1}^{\prime}$ is a $v$-colorful path and the proof is complete.

\section{Long Rainbow Paths in $\chi(G)$-Colorings}

The following theorem shows that for every graph $G$ with $\chi(G)=\omega(G)$, the conjecture is true.

Theorem 5 Let $G$ be a graph with $\omega(G)=\chi(G)$. Then there exists a $\chi(G)$-coloring of $G$ with a v-colorful path for every $v \in V(G)$.

Proof. Assume that $M=\left\{v_{1}, \ldots, v_{\chi(G)}\right\}$ is a maximum clique in $G$. We claim that the assertion holds for the coloring $f=\mathcal{C}(G, M, c)$, where $c$ is an arbitrary coloring of $G$. By Lemma 2, for every $v \in V(G)$, there exists a directed path in $G_{f}$, starting from $v$ and ending in $M$. Call this path by $P: p_{1}, \ldots, p_{k}$. Let $M^{\prime}=\left\{u_{1}, \ldots, u_{\chi(G)-k}\right\}$ be a subset of $M$ such that for every $j, 1 \leq j \leq \chi(G)-k, c\left(u_{j}\right) \notin\left\{c\left(p_{1}\right), \ldots, c\left(p_{k}\right)\right\}$. Clearly, $p_{1}, \ldots, p_{k}, u_{1}, \ldots, u_{\chi(G)-k}$ is a $v$-colorful path. 
In the previous theorems, we proved the existence of $v$-colorful paths (rainbow paths of length $\chi(G)$ ), for every $v \in V(G)$, using a set of colors with different sizes. We close this paper by showing that there are some $\chi$-colorings of $G$ in which there exist long $v$-rainbow paths, for every $v \in V(G)$.

Theorem 6 Let $G$ be a connected graph. Then there is a $\chi(G)$-coloring of $G$ in which for every $v \in V(G)$, there exists a $v$-rainbow path of length $\left\lfloor\frac{\chi(G)}{2}\right\rfloor$.

Proof. Let $c$ be a $\chi$-coloring of $G$. As a consequence of Proposition 5 in [3], there is a path $P: p_{0}, \ldots, p_{\chi(G)-1}$ such that

$$
c\left(p_{i}\right)= \begin{cases}i & \text { if } 0 \leq i \leq m \\ \chi(G)+m-i & \text { if } m+1 \leq i \leq \chi(G)-1\end{cases}
$$

where $m=\left\lfloor\frac{\chi(G)-1}{2}\right\rfloor$. Let $c^{\prime}=\mathcal{C}(G, P, c)$. By Lemma 2 for every $v \in V(G)$, there is a path $Q(v): v=q_{1}, \ldots, q_{k}=p_{s}$, where $c^{\prime}\left(q_{i+1}\right)=c^{\prime}\left(q_{i}\right)+1(\bmod \chi(G))$ for $1 \leq i<k$. With no loss of generality, assume that $q_{k} \in V(P)$ and $q_{i} \notin V(P)$ for each $i, 1 \leq i \leq k-1$. Let $Q^{\prime}(v): q_{1}^{\prime}, \ldots, q_{k+\left\lfloor\frac{\chi(G)}{2}\right\rfloor}^{\prime}$ be the path of length $k+\left\lfloor\frac{\chi(G)}{2}\right\rfloor-1$ such that

$$
q_{i}^{\prime}= \begin{cases}q_{i} & \text { if } 1 \leq i \leq k \\ p_{s+(i-k)} & \text { if } k+1 \leq i \leq k+\left\lfloor\frac{\chi(G)}{2}\right\rfloor \text { and } s \leq m \\ p_{s-(i-k)} & \text { if } k+1 \leq i \leq k+\left\lfloor\frac{\chi(G)}{2}\right\rfloor \text { and } m<s .\end{cases}
$$

We claim that the first $\left\lfloor\frac{\chi(G)}{2}\right\rfloor+1$ vertices of $Q^{\prime}(v)$ form a $v$-rainbow path. We prove this in the case $s \leq m$. The other case $(s>m)$ is similar.

Let $t$ be the integer that $q_{t}^{\prime}=p_{m}$. If $t \geq\left\lfloor\frac{\chi(G)}{2}\right\rfloor+1$, then it is clear that there is a $v$-rainbow path of length $\left\lfloor\frac{\chi(G)}{2}\right\rfloor$. Thus assume that $t \leq\left\lfloor\frac{\chi(G)}{2}\right\rfloor$. We have

- $c^{\prime}\left(q_{i+1}^{\prime}\right)=c^{\prime}\left(q_{i}^{\prime}\right)+1$, for $i, 1 \leq i<t$; and

- $c^{\prime}\left(q_{i}^{\prime}\right)=c^{\prime}\left(q_{i+1}^{\prime}\right)+1$, for $i, t+1 \leq i \leq\left\lfloor\frac{\chi(G)}{2}\right\rfloor$.

Therefore, $c^{\prime}\left(q_{i}^{\prime}\right) \in\{0, \ldots, m\}$ for $i, 1 \leq i \leq t$, and $c^{\prime}\left(q_{i}^{\prime}\right) \in\{m+1, \ldots, \chi(G)-1\}$ for $i$, $t+1 \leq i \leq\left\lfloor\frac{\chi(G)}{2}\right\rfloor+1$. Hence the color of the vertices of $q_{1}^{\prime}, \ldots, q_{\left\lfloor\frac{\chi(G)}{2}\right\rfloor+1}^{\prime}$ are distinct and this path is a $v$-rainbow path.

Acknowledgments. The authors wish to express their deep gratitude to the referee of the paper for making valuable suggestions. The research of the first author was in part supported by a grant from IPM (No. 89050212). 


\section{References}

[1] S. Akbari, F. Khaghanpoor, S. Moazzeni, Colorful paths in vertex coloring of graphs, submitted.

[2] J.A. Bondy, U.S.R. Murty, Graph Theory, Graduate Texts in Mathematics, 244. Springer, New York, 2008.

[3] D. de Werra and P. Hansen, Variations on the Roy-Gallai Theorem, 4OR 3 (2005) 245-251.

[4] T.S. Fung, A colorful path, The Mathematical Gazette 73 (1989) 186-188.

[5] H. Li, A generalization of the Gallai-Roy theorem, Graphs and Combinatorics 17 (2001) 681-685.

[6] C. Lin, Simple proofs of results on paths representing all colors in proper vertexcolorings, Graph and Combinatorics 23 (2007) 201-203. 